\title{
Metacognição como proposta pedagógica nos cursos de Terapia Ocupacional: priorização do raciocínio clínico
}

\author{
Metacognition as pedagogical proposal in Occupational Therapy \\ courses: prioritization of reasoning
}

\author{
1 Márcia Regina de Assis marcia.assis@ifrj.edu.br \\ 1 Mauricio Abreu Pinto Peixoto \\ 1 Pedro Henrique Maraglia \\ 2 Leonardo Valesi Valente \\ 3 Marcos Antônio Gomes Brandão \\ 1 Universidade Federal do Rio de Janeiro - Núcleo de Tecnologia Educacional para a Saúde.
2 Instituto Federal de Educação, Ciência e Tecnologia do Rio de Janeiro.
3 Universidade Federal do Rio de Janeiro - Escola de Enfermagem Anna Nery.
}

\section{Resumo}

O raciocínio clínico é importante para a caracterização do domínio da prática em Terapia Ocupacional (TO), mas o seu aprendizado é difícil, em função de sua estrutura complexa que, ao incluir as dimensões científica, narrativa, pragmática e ética, implica na execução de variadas operações mentais pelo aluno no seu aprendizado e pelo profissional na sua atuação cotidiana. Este artigo objetiva apresentar a metacognição como recurso auxiliar para o ensino do raciocínio clínico em Terapia Ocupacional. Realizou-se estudo metodológico fundamentado em revisão narrativa que busca estabelecer interface entre o ensino do raciocínio clínico em TO e as bases teóricas e conceituais do domínio metacognitivo e da aprendizagem significativa. Percebe-se a necessidade de reflexão e pensamento crítico para fazer diagnóstico clínico em TO. A metacognição caracteriza-se pelo seu foco dirigido a esses processos e por sua capacidade de explicitá-los e de permitir o seu gerenciamento. No entanto na Terapia Ocupacional, o uso da metacognição ainda não faz parte das propostas de ensino-aprendizagem do raciocínio clínico. Dado o seu desempenho positivo em domínios similares, conclui-se ser factível o uso da metacognição para facilitar o ensino e o aprendizado do raciocínio clínico em TO.

\section{Palavras chave}

Metacognição. Raciocínio clínico. Terapia Ocupacional.

\begin{abstract}
Clinical reasoning is important for the characterization of the field of practice in Occupational Therapy (OT), but its learning is difficult due to its complex structure which, by including the scientific, narrative, pragmatic and ethical dimensions, implies the execution of varied mental operations by the student in their learning and by the professional in their daily performance. This article aims to present metacognition as an auxiliary resource for teaching clinical reasoning in Occupational Therapy. A methodological study was carried out based on a narrative review that seeks to establish an interface between the teaching of clinical reasoning in OT and the theoretical and conceptual bases of the metacognitive domain and meaningful learning. It is noticed the need for reflection and critical thinking to make a clinical diagnosis in OT. Metacognition is characterized by its focus on these processes and its ability to make them explicit and enable them to be managed. However, in Occupational Therapy, the use of metacognition is not yet part of the teaching-learning proposals of clinical reasoning. Given its positive performance in similar domains, it is concluded that the use of metacognition is feasible to facilitate the teaching and learning of clinical reasoning in OT.
\end{abstract}

\section{Keywords}

Metacognition. Clinical reasoning. Occupational Therapy.

\section{Como você deve citar?}

ASSIS, Márcia Regina de et al. Metacognição como proposta pedagógica nos cursos de Terapia Ocupacional: priorização do raciocínio clínico. Cadernos UniFOA, Volta Redonda, n. 36, p. 79-89, abr. 2018. 


\section{INTRODUÇÃO}

O cuidado em saúde pode ser entendido como "o tratar, o respeitar, o acolher, o atender o ser humano em seu sofrimento - em grande medida fruto de sua fragilidade social, mas com qualidade e resolutividade de seus problemas" (PINHEIRO, 2009). A resolutividade de problemas na área da saúde usualmente é dependente do diagnóstico e da intervenção clínica, independentemente da especialidade de cada profissional que presta o cuidado. Para que a resolução de problemas ocorra por meio da aplicação do raciocínio clínico e seja efetiva, antes de tudo, é necessária uma qualificada capacidade de pensar e refletir. $O$ desenvolvimento dessa capacidade de pensamento e reflexão a ser expressa no raciocínio clínico carece de estratégias de aprendizagem que explorem ao máximo as potencialidades da cognição do indivíduo. Como veremos adiante, esse processo de reflexão beneficia-se do emprego de processos de controle e monitoramento que são relacionados à metacognição.

O objetivo deste artigo é apresentar a metacognição como recurso auxiliar para o ensino do raciocínio clínico em Terapia Ocupacional. Para tanto, utiliza como método o estudo metodológico fundamentado em revisão narrativa que busca estabelecer interface entre o ensino do raciocínio clínico em TO e as bases teóricas e conceituais do domínio metacognitivo e da aprendizagem significativa. 0 estudo foi organizado em três tópicos. 0 primeiro problematiza e justifica a razão de ser para o desenvolvimento do estudo. 0 segundo apresenta a metacognição nos aspectos pertinentes ao problema. Por fim, o terceiro tópico une os dois anteriores, orientando-se para fomentar uma discussão dirigida visando a melhor formação do aluno de graduação em Terapia Ocupacional.

\section{O PROBLEMA: RACIOCÍNIO CLÍNICO E O CONCEITO DE OCUPAÇÃO}

\subsection{Raciocínio Clínico}

Em termos genéricos, o raciocínio clínico é o processo usado por profissionais para refletir, planejar, orientar e conduzir o tratamento do paciente (SCHELL, 2002). Este estudo abordará especificamente a importância do raciocínio clínico para o curso de Terapia Ocupacional (TO). Para o terapeuta ocupacional, é um processo contínuo, que começa quando o profissional lê o encaminhamento do paciente, e se estende pela avaliação, intervenção e alta. Ele é um elemento importante na caracterização do domínio da prática em Terapia Ocupacional (HOOPER, 1997 apud MANCINI, 2008). É tema central no ensino da TO, dada a ênfase clínica da profissão, que é voltada aos cuidados no cotidiano dos seus pacientes.

O raciocínio clínico em Terapia Ocupacional ainda é um tema pouco estudado no Brasil, com poucos livros-texto, traduções ou pesquisas na área (MARCOLINO, 2014). A autora em seu estudo aponta que, nos moldes educacionais praticados no Brasil, ainda faltam propostas de pesquisa que busquem compreender processos de apropriação de modos mais complexos de raciocínio durante a formação inicial.

Carrie et al. (2010), em revisão narrativa, identificaram na literatura que o raciocínio clínico depende de um conjunto de variáveis, distribuídas em cinco categorias: 1) processos cognitivos; 2) dimensões do raciocínio; 3) fatores moduladores; 4) métodos de documentação e; 5) aspectos do contexto do atendimento. No escopo deste estudo, interessa-nos a primeira. Para os autores, o termo "fatores cognitivos" engloba uma expressão geral que se refere ao processo de resolução de problemas, que é tratado na perspectiva do reconhecimento de padrões. Por consequência, importa nesse domínio explorar a percepção do problema, identificação de sinais diagnósticos, formulação do problema pelo uso de esquemas fundamentados no conhecimento e experiência anteriores e finalmente a identificação de soluções potenciais. Os autores ressaltam que esses fatores estão ainda pouco explorados e, por isso, 
não se sabe o papel exato que eles têm na construção do diagnóstico e da terapêutica em TO. Entretanto, enfatizam que, na medida em que esses fatores moldam a maneira como os terapeutas ocupacionais pensam e atuam, eles podem ter influência direta sobre os serviços que prestam aos seus clientes.

O diagnóstico, sendo um elemento do raciocínio clínico em TO, é complexo. Para realização da avaliação e tratamento, os terapeutas ocupacionais empregam simultaneamente pelo menos 4 linhas de raciocínio clínico. E essas linhas implicam nas variadas operações mentais que os terapeutas ocupacionais precisam usar, quando trabalham com seus pacientes, constituindo assim uma estrutura específica de pensamento da Terapia Ocupacional (NEISTADT, 1998 apud MANCINI, 2008). São elas (MANCINI, 2008; RADOMSKI, 2013):

Científica - refere-se ao pensamento lógico sobre a natureza dos problemas do paciente e a forma de selecionar as teorias e procedimentos que serão importantes para guiar a decisão clínica;

Narrativa - é assim denominada porque compreende a história ocupacional do cliente (história de vida, atividades preferidas, hábitos, rotinas e papeis) e o significado pessoal de determinada condição de saúde, buscando definir como o cliente vivencia a experiência diária com essa condição de saúde;

Pragmática - relativa aos aspectos práticos da prestação de serviços, ou seja, ao contexto mais amplo da prática clínica. Considera os fatores limitantes e facilitadores relacionados ao implemento da intervenção. Esses fatores incluem o ambiente de tratamento, os recursos financeiros e sociais da instituição e do paciente, conhecimentos, habilidades e experiências do terapeuta, entre outros;

Ética - envolve a ponderação de riscos e benefícios para o cliente e estabelece prioridades e determina o curso de ações que atendam as demandas dos clientes e que sejam consistentes clinicamente. Esse tipo de raciocínio tem sido identificado como elemento chave da tomada de decisão clínica.

Essa multidimensionalidade e simultaneidade da estrutura de pensamento da TO exige um grande esforço cognitivo dos alunos da graduação, isto é, a cada contexto clínico, os alunos têm que buscar o conhecimento adquirido nas diversas disciplinas, ouvir o relato dos pacientes, ler os prontuários para poderem tomar a decisão mais adequada e adaptar-se a objetivos dinâmicos, conforme cada tipo de atuação, e isso, ressalte-se, em um contexto em que nenhuma das habilidades necessárias para tal está plenamente desenvolvida. Ainda mais, precisam buscar em suas próprias histórias de vida variados recursos para interpretar os dados disponíveis, atribuindo-lhes o significado particular e específico a cada situação da prática profissional, que não é uma tarefa fácil, para quem está em processo de formação, cujo desenvolvimento marcado pelas experiências da graduação se estende pela formação continuada em programas adicionais de formação complementar, residências, especializações e supervisão profissional.

\section{CONCEITO DE OCUPAÇÃO E A APRENDIZAGEM SIGNIFICATIVA}

Outro fator que dificulta o processo de raciocínio clínico dos alunos é o significado, muitas vezes equivocado, que atribuem ao termo "ocupação". Na experiência de um dos autores, docente em TO, é frequente que o termo seja entendido sob o viés do senso comum, ou seja, a "ocupação" interpretada como sinônimo de "passatempo", "lazer" ou "entretenimento". A Língua Portuguesa contribui para isso, oferecendo diversos significados distintos daquele que é de fato proposto pela TO. Para a Terapia Ocupacional, o termo ocupação deve ser compreendido "[...] como um modo ativo de o paciente intervir no mundo e, assim, ativamente, estar consigo e com os outros" (JORGE, 1990, p.19). Assim, o termo é amplo e complexo. É construído a partir de modelos teórico-filosóficos do estudo da natureza da ocu- 
pação humana que qualifica os comportamentos, as interações entre os seres, os objetos, os ambientes e a capacidade de transformação requerida nessas relações, dando destaque a um comportamento ocupacional observável tanto na performance diária do cotidiano, quanto objetivamente mensurável no desempenho ocupacional de cada paciente assistido (KIELHOFNER, BURKE, 1980; KIELHOFNER, HENRY, WALENS, 1989; KIELHOFNER, 1995). A dificuldade do aluno em discriminar os múltiplos significados atribuídos pelo senso comum daquele específico para a profissão pode favorecer o aprendizado insatisfatório do raciocínio clínico. Dessa forma, a tomada de decisão baseada em um conceito equivocado determinará condutas inadequadas e sem efetividade para a assistência do referido paciente.

De modo análogo, a supervisão prática de estagiários, em formação nos cursos brasileiros de graduação em Terapia Ocupacional, envolve preocupação com o raciocínio clínico que apoia a capacidade avaliativa eficiente, a tomada de decisão, o gerenciamento de respostas adaptativas dos pacientes e os manejos incessantes de quais objetivos priorizar para a efetivação da assistência terapêutica ocupacional em diversos contextos prático-clínicos (GUIMARÃES, FALCÃO, 2004; DAHDAH, FRIZZO, FANGEL, 2014; RUAS et. al., 2015; ANVERSA, BORGES, 2016).

O estudo de Fornazieiro et al. (2011), com alunos do curso de Medicina, apontou que, quando questionados sobre seu aprendizado do raciocínio clínico, relataram dificuldades em exercê-lo. Para eles, o grande problema foi a incerteza sobre a correção dos procedimentos a serem aplicados na rotina clínica. Os autores entenderam essa dificuldade à luz da teoria da aprendizagem significativa. Essa insegurança decorria da dificuldade de fundamentá-los, isto é, faziam diagnósticos sem a capacidade de explicitar o conjunto de conhecimentos e a maneira de conectá-los, de modo a chegar ao diagnóstico. Por consequência, apontaram essa teoria e seus desdobramentos como alternativa possível, para superar essas dificuldades, dado o relacionamento que promove entre o procedimento em tela e o conjunto de conhecimentos que o fundamenta.

Entende-se que a aprendizagem significativa (AUSUBEL, 2000) pode ser uma alternativa para minimizar as dificuldades vivenciadas pelos alunos na aprendizagem do raciocínio clínico, cabendo construir os elementos que possam explicitar como esse processo pode ocorrer.

Da Teoria da Subsunção, desenvolvida por David Ausubel, na década de 1960, extraímos o conceito de Aprendizagem Significativa, que é relevante para o tema que aqui se apresenta. Nessa teoria, o aprendizado se dá pela subsunção (ou subordinação) lógica de um conceito mais restrito e concreto a outro mais amplo e abstrato. Para esse autor, o conhecimento é significativo por definição e envolve essa relação de subordinação (AUSUBEL, 2000).

A teoria de Ausubel preconiza a estruturação cognitiva como prioritária na aprendizagem, em relação interdependente com os demais aspectos - psicomotores e afetivos (SALVADOR et al., 2014). Seu campo de estudo prioritário foi o ambiente tradicional de sala de aula. Nesse contexto, afirmou que a aprendizagem se dá na mente do aluno, por estruturação dos conteúdos em uma trama cognitiva. Essa rede de conteúdos se organiza de forma hierárquica, com os conceitos mais abrangentes subordinando os mais específicos. Se ao aprender o aluno modifica essa rede, caracteriza-se a aprendizagem significativa.

Aqui cada novo conteúdo é agregado à trama, respeitando-se as relações de subordinação. Nesse caso, o aprendizado é significativo no sentido de que o novo se integra ao antigo, produzindo novos significados, já que estes se expressam pelo tipo de relação que os conceitos estabelecem entre si. Nesse sentido, então, cada nova aprendizagem reorganiza a trama na sua totalidade. 
Entretanto, cabe aqui enfatizar que aprender significativamente não é, necessariamente, aprender os conteúdos corretos. Significa apenas que houve subordinação lógica de um conceito novo a um pré-existente. Isso é importante neste texto, porque como dissemos antes, uma das dificuldades com o raciocínio clínico em TO é o conceito prévio do aluno sobre "ocupação".

Se durante a graduação o sistema de ensino não for capaz de romper com a ideia de ocupação como passatempo, é possível que todo o conteúdo do curso seja aprendido de forma subordinada ao conceito prévio equivocado. Como consequência, é possível que muitos egressos tomem como objetivo profissional meramente entreter os pacientes. Nesse caso, então, todo o aprendizado serve apenas para adquirir um conjunto de ferramentas, visando ao lazer do paciente, entendido no seu sentido mais simplório. Por isso, os cursos de graduação em Terapia Ocupacional correm o risco de não atingir seu objetivo. Ao invés de formar profissionais capazes de aplicar, ética e adequadamente, os conceitos e técnicas da profissão, terminam por formar profissionais que se preocupam exclusivamente em combater o ócio de seus clientes.

O uso reflexivo do raciocínio clínico requer estímulo ao desenvolvimento da estrutura cognitiva dos alunos (SCHELL, 2002; JACOBS, JACOBS, 2006). Nesse sentido, é que podemos entender a importância de que o aluno seja capaz de ressignificar seus conceitos anteriores, de ocupação, cotidiano, funções, atividades produtivas, lazer e autocuidado. É importante que esses conceitos, originalmente baseados no senso comum, passem a compatibilizar-se com o que postula a Associação Americana de Terapia Ocupacional (AOTA, 2015).

Por isso, é preciso modificar o conceito reducionista de Terapia Ocupacional como "lazer", "combate ao ócio" pelo de uma profissão que tem o "[...] propósito de melhorar ou possibilitar a participação em papéis, hábitos e rotinas em diversos ambientes como casa, escola, local de trabalho, comunidade e outros lugares." (AOTA, 2015, p.1). E aqui, novamente, o conceito de ocupação precisa modificar-se de mero passatempo para o de uma atividade com metas e objetivos e, portanto, plena de significados. Mais que isso, atribuir-Ihe os significados compatíveis com o corpo de conhecimentos que fundamenta a TO, fornecendo-Ihe a identidade e discriminando-a de outras atividades que lhe possam assemelhar-se.

Entretanto não é fácil, nem simples tal tarefa, desenvolver raciocínio clínico durante a formação a partir da aprendizagem significativa. Não basta à instituição escolar apresentar ao aluno os novos conceitos. Cabe lembrar que a mera apresentação pode levar à "decoreba" e aos seus efeitos danosos. É preciso que o aluno passe a atribuir à TO o significado que aqui apresentamos. Caso contrário, esses egressos poderão agir aparentemente como terapeutas ocupacionais, quando, na verdade, estarão apenas atenuando o ócio dos seus pacientes. Para isso, é necessário que haja um contexto pedagógico onde essa mudança seja possível. Importa criar condições para que o conceito de ocupação seja ressignificado. A metacognição é uma ferramenta de ensino/aprendizagem que pode auxiliar também nisso, ao favorecer aumento da consciência e gerência dos processos cognitivos do aluno.

\section{RESULTADOS E DISCUSSÃO}

\subsection{Metacognição}

O termo metacognição foi definido por John Flavell, na década de 1970, como sendo um discurso de segundo nível sobre a cognição, uma cognição sobre a cognição (FLAVELL, 1970). Para Peixoto, Brandão e Santos (2007, p.70), a 
metacognição é um termo amplo, usado para descrever diferentes aspectos do conhecimento que construímos sobre como nós percebemos, recordamos, pensamos e agimos. Uma capacidade de saber sobre o que sabemos. Um pensamento sobre o pensamento, uma cognição sobre a cognição ou um atributo cognitivo ou conhecimento sobre o fenômeno cognitivo.

Por isso, o indivíduo, ao refletir sobre seu próprio pensamento, tem a oportunidade de aprimorar o seu desempenho cognitivo perante os desafios propostos.

Segundo Ribeiro (2003), há duas formas principais de compreensão da metacognição: a primeira é o conhecimento sobre o conhecimento, ou seja, a tomada de consciência dos processos e das competências necessárias para a realização da tarefa. A segunda é o controle ou autorregulação, que é a capacidade para avaliar a execução da tarefa e fazer correções, quando necessário.

Neves (2007) afirma que a aprendizagem é uma ação metacognitiva, uma vez que é um processo consciente que transforma em conhecimento as informações adquiridas. Para a autora, o uso da cognição e da metacognição proporciona o armazenamento de elementos da informação ou representação de um determinado fato. Uma vez reunidos, esses elementos são tomados como base para a conversão de novas ideias, possibilitando assim, a transformação da ideia original em conhecimento integrado a nossa mente.

De acordo com Ribeiro (2003), a aprendizagem na perspectiva metacognitiva apresenta alguns benefícios, a saber: a autoapreciação e o autocontrole cognitivos como formas de pensamento que 0 aprendiz pode desenvolver e que lhe permite ter um papel ativo em seu próprio conhecimento. A metacognição destaca o papel pessoal na avaliação e controle cognitivos dos sujeitos que, com idênticas capacidades intelectuais, podem ter diferentes níveis desempenho, devido à forma como cada um atua sobre os seus próprios processos de aprendizagem. Por isso, a metacognição permite ao aprendiz avançar no seu nível de realização.

Ainda segundo essa autora, a eficácia da aprendizagem não depende apenas da idade, experiência e nível intelectual. Depende ainda da aquisição de estratégias cognitivas e metacognitivas que possibilitem ao sujeito planejar e monitorar o seu desempenho, ou seja, a metacognição permite a tomada de consciência dos processos utilizados para aprender e também a tomada de decisões adequadas sobre quais estratégias utilizar em cada tarefa e, ainda, avaliar a sua validade, fazendo as modificações necessárias, quando não se alcança os resultados esperados.

Por isso, a metacognição pode ser entendida como uma capacidade chave de que depende a aprendizagem: aprender a aprender, o que é um requisito muito importante, para que os alunos e futuros terapeutas ocupacionais possam afastar-se do senso comum e entender ocupação de maneira mais profissional e técnica.

\section{METACOGNIÇÃO COMO PROPOSTA PEDAGÓGICA PARA A APRENDIZAGEM DO RACIOCÍNIO CLÍNICO}

Em Terapia Ocupacional, o uso da metacognição ainda não faz parte das propostas de ensino-aprendizagem do raciocínio clínico. Todavia em outras áreas da saúde, como a enfermagem e a medicina, a apropriação da metacognição tem demonstrado resultados interessantes, como, por exemplo, os estudos sobre metacognição e o ensino de enfermagem de: Stedlie e Friendlander (2003), os estudos de Silva et al. (2011), Araújo e Makowsk (2014), Brandão e Peixoto (2014) e Silva, Santos e Vargens (2015), sobre metacognição e o diagnóstico de Enfermagem. Na Medicina, assim como na Enfermagem, há estudos relevantes no uso da metacognição na formação dos médicos, como, por 
exemplo: Peixoto et al. (2007), Sand (2009) e Eichbaum (2014). De forma geral, esses estudos mostram que a metacognição na Medicina e Enfermagem colabora no aprendizado tanto do diagnóstico quanto da terapêutica. Dada a ampla interface entre essas áreas e a TO, é razoável inferir que também aqui, a metacognição pode contribuir para a formação dos futuros terapeutas ocupacionais, por meio do estímulo ao autoconhecimento e à maior capacidade crítica e reflexiva. Nesse sentido, a metacognição pode ser entendida como uma tecnologia educacional simbólica (PEIXOTO; BRANDÃO; SANTOS, 2007), isto é, uma ferramenta de desenvolvimento e manutenção em operação do sistema de pensamento e aprendizado humanos.

O processo de construção do raciocínio clínico, como dito anteriormente, exige reflexão e pensamento crítico do aluno de graduação em Terapia Ocupacional. Porém, como vimos, os conceitos previamente internalizados (de ocupação, por exemplo) na estrutura cognitiva do aprendiz costumam contrapor-se aos novos conceitos necessários para a realização adequada da terapia ocupacional. Por isso, há a necessidade de consciência, monitoração e controle sobre essas operações mentais, que fazem parte do processo metacognitivo.

Além do papel do aluno no contexto de aprendizagem do raciocínio clínico, o professor também tem papel fundamental, pois cabe a ele mediar a relação do aluno com seu conhecimento prévio e relevante. Assim, para a aprendizagem significativa do raciocínio clínico, ele deverá trazer à tona aquilo que poderá servir como âncora, para a construção de novos conhecimentos potencialmente significativos (SANTOS, 2006). Ao professor, cabe também mediar o processo de reflexão e pensamento crítico, quando o conhecimento prévio não for relevante, inadequado ou incorreto. Quando se pensa no processo de ensino, a metacognição pode ajudar o aluno na monitoração dos processos de compreensão e redação, que o auxiliam na sua percepção sobre essas tarefas, melhorando assim o seu aprendizado (JOU; SPERB, 2006; DAMIANI et al., 2006; ANDREATTA, 2010).

Nessa perspectiva, vale considerar, por exemplo, que, durante o processo de construção do raciocínio clínico, a monitoração do processo de compreensão pode ser realizada por meio da solicitação ao aprendiz, para que expresse em voz alta suas ações. Isso o ajudará a compreender a maneira de raciocinar clinicamente, assim como perceber eventuais erros na condução da tarefa.

$\mathrm{Na}$ Terapia Ocupacional, em especial, essa solicitação pode trazer subsídios para a percepção de verificar se o aluno está seguindo os conceitos próprios da profissão ou aqueles do senso comum, que não corresponderão ao adequado aprendizado do raciocínio clínico, fundamental para o cuidado aos indivíduos que necessitam da Terapia Ocupacional.

O raciocínio clínico é um importante recurso para o ato de cuidar e o seu aprendizado faz parte da formação dos alunos, tanto do curso de graduação em Terapia Ocupacional, como também de todos os demais alunos dos cursos de graduação em Ciências da Saúde. Segundo Côrrea (2003, p.151), "cuidar é responder ao contínuo convite para decidir." Ainda segundo a autora, ao ter consciência desse convite, o profissional se vê diante do desafio de enfrentar as dúvidas, incertezas e subjetividades inerentes aos julgamentos que norteiam suas decisões, que envolvem processos mentais complexos que requerem conhecimento de seu conteúdo e procedimentos. E por isso, a metacognição como ferramenta, para aprendizagem do raciocínio clínico, amplia o contexto de ensino ao favorecer a monitoração, a análise, o planejamento, a avaliação e a reavaliação, que facilitam o desenvolvimento da autorregulação crítica e reflexiva do pensamento.

A metacognição também tem relevante valor para o aluno, em seus esforços para um aprendizado autônomo e significativo (DAMIANI et al., 2006; ANDREATTA et al., 2010), pois, colabora com a perspectiva histórico-social da tecnologia educacional em sua ênfase no pensamento crítico, ao 
estimular a consciência, a reflexão e a gerência dos processos cognitivos. Nesse sentido, favorece a autonomia do aluno, na medida em que busca valorizar o papel dele, no binômio ensino-aprendizagem (SILVA; PEIXOTO, 2015).

Para Santos (2006), a metacognição, como uma tecnologia educacional, deve ser explorada. $\mathrm{E}$, para isso, segundo a autora, é preciso investigar, inicialmente, qual o conhecimento metacognitivo que o aprendiz possui e, a partir daí, estimular nele uma atitude metacognitiva, a fim de proporcionar o desenvolvimento de habilidades metacognitivas, com as quais ele poderá regular seu pensamento crítico e ativo na construção do seu conhecimento. E, principalmente, que esse conhecimento venha a capacitá-lo para o exercício da profissão, no cuidado integral aos indivíduos.

\section{CONSIDERAÇÕES FINAIS}

O raciocínio clínico envolve variados aspectos cognitivos, entretanto, é importante que seja dito, envolve ainda variadas interpretações idiossincrásicas dependentes de fatores psicológicos, afetivos, culturais, sociais e políticos. Somos seres imersos e em constante relação com o mundo. No dizer de Ortega y Gasset (1967, p. 52), "eu sou eu e minha circunstância, e se não salvo a ela, não salvo a mim". O aluno em formação dimensiona sua aprendizagem para abarcar as competências capazes de responder às incessantes demandas que emergem no contato com as ocupações e os cotidianos de futuros assistidos nos mais complexos contextos da atuação clínica.

A metacognição envolve em seu aspecto mais fundamental uma resistência à alienação; uma chamada à consciência. Isso é fundamental, pois raciocinar clinicamente é ser capaz de tomar decisões que auxiliarão pessoas a promover, prevenir, reabilitar ou recuperar sua saúde. Acreditamos que esse desenvolvimento de consciência mereça orientar toda a formação, amparando a possibilidade de superação pessoal do aprendiz em direção ao futuro profissional.

O processo de raciocínio clínico é importante elemento na formação dos alunos da graduação na área da saúde. Em Terapia Ocupacional, não é diferente. Por isso, é fundamental que haja investimento no processo de ensino-aprendizagem durante todo o período de formação, considerando-se a complexidade do raciocínio clínico. 0 processo ensino-aprendizagem será mais eficaz, se for fundamentado na importância de ensinar a pensar e a formar futuros profissionais reflexivos e críticos. Ressalte-se aqui que a deformação produzida pela ausência de reflexão gera atuações profissionais distorcidas, dificilmente reconhecíveis pelo profissional em ação, levando a sua perpetuação. Nesse sentido, a chamada à consciência favorecida pelo uso adequado da metacognição é fator importante em apoio à reflexão e crítica necessárias ao bom profissional. Aprendizes da profissão devem submeter-se a processos incessantes de articulação entre os saberes prévios e os atuais. É importante que atualizem seus próprios conceitos, para que, em sintonia com o corpo de conhecimentos da TO, possam de forma adequada, oferecer o legítimo cuidado profissional às pessoas que dele necessitem. $O$ uso metacognição, dado o seu desempenho positivo em tarefas em domínios similares, permite concluir que é factível para facilitar o ensino e o aprendizado do raciocínio clínico em TO. No entanto, dados os limites deste estudo teórico, cabe enfatizar a necessidade de validação empírica das proposições aqui apresentadas. 


\section{REFERÊNCIAS}

AMERICAN OCCUPATIONAL THERAPY ASSOCIATION (AOTA). Estrutura da prática da Terapia Ocupacional: domínio \& processo $3^{a}$ ed. Rev Ter Ocup Univ São Paulo, v. 26, 2015 (ed. esp.), p.1-49. Traduzido para o português por Alessandra Cavalcanti et al.

ANDREATTA, I.; SILVA, J. G.; SUSIN, N.; FREIRE, S. D. Metacognição e Aprendizagem: como se relacionam? PSiCo, Porto Alegre, PUCRS, v. 41, n. 1, 2010, p. 7-13.

ANVERSA, A. C.; BORGES, J. M. Prática de estágio em terapia ocupacional na comunidade. Cad. Ter. Ocup. UFSCar, v. 24, n. 4, p. 821-826, 2016.

ARAÚJO, K. S.; MAKOWSK, R. M. Estratégias de ensino aprendizagem no curso de enfermagem: a construção do saber clínico norteado pela saúde humana multidisciplinar. Colóquio Internacional de Educação 2014. Universidade do Oeste de Santa Catarina, 2014, P. 1094-1102.

AUSUBEL, D. P. Aquisição e retenção de conhecimentos: uma perspectiva cognitiva. Lisboa: Paralelo Editora, LTDA, 2000.

BRANDÃO, M. A. G.; PEIXOTO, M. A. P. Estratégias Metacognitivas para o ensino e aprendizagem de diagnósticos de enfermagem. In: HERDMAM, T. H.; NAPOLEÃO, A. M. A.; LOPES, C. T.; SILVA, V. M. (Org.). PRONANDA - Programa de Atualização em Diagnósticos de Enfermagem: Ciclo 4.ed. Porto Alegre: Artmed Panamericana, v. 2, 2016 p. 87-130.

CARRIER, A.; LEVASSEUR, M.; BE DARD, D.; DESROSIERS, J. Community occupational therapists' clinical reasoning: Identifying tacit knowledge. Australian Occupational Therapy Journal, v.57, 2010, p. 356-365.

CORRÊA, C. G. Raciocínio clínico: o desafio do cuidar. Tese (Doutorado em Enfermagem). Escola de Enfermagem. Universidade de São Paulo, 2003. 175p.

DAHDAH, D. F.; FRIZZO, H. C. F.; FANGEL, L. M. V. Terapia ocupacional em contextos hospitalares caracterização do ensino nos cursos de graduação universitários brasileiros. Rev Ter Ocup Univ São Paulo, v. 25 n. 1, p. 70-9, 2014

DAMIANI, M. F.; GIL, R. L.; PROTÁSIO , M. R. A metacognição como auxiliar no processo de formação de professoras: uma experiência pedagógica. UNIrevista, v.1, n 2, 2006, p. 1-14.

EICHBAUM, Q. G. Thinking about Thinking and Emotion: The Metacognitive Approach to the Medical Humanities that Integrates the Humanities with the Basic and Clinical Sciences. Perm J., v. 18, n.4, 2014, p.64-75.

FLAVELL, J.H. Metacognition and cognition monitoring: a new area of cognitive-developmental inquiry. American Psychologist, v.34, n.10, 1979, p. 906-911.

FORNAZIERO, C. C. et al. O Processo de Ensino e Aprendizagem do Raciocínio Clínico pelos Estudantes de Medicina. Revista Brasileira de Educação Médica, v. 35, n. 2, 2011, p. 246 - 253

GUIMARÃES, D. S. L.; FALCÃO, I. V. Análise de atividades e formação do terapeuta ocupacional: um estudo com os preceptores de estágio da UFPE. Rev. Ter. Ocup. Univ. São Paulo, v. 15, n. 2, p. 63-70, 2004. 
JACOBS, K.; JACOBS, L. Dicionário de Terapia Ocupacional - Guia de referência. São Paulo: ROCA, 2006, 4ed, 248p.

JORGE, R. C. 0 objeto e a especificidade da Terapia Ocupacional. Belo Horizonte: Gesto, 1990, 95p.

JOU, G. I.; SPERB, T. M. A Metacognição como Estratégia Reguladora da Aprendizagem. Psicologia: Reflexão e Crítica, v.19, n. 2, 2006, p. 177-185.

KIELHOFNER, G. A model of human occupation: theory and application. Baltimore, MD: Williams \& Wilkins, 1995, 565p.

KIELHOFNER, G.; BURKE, J. P. A model of human occupation, part 1. Conceptual framework and content. Am J Occup Ther., v. 34, n. 9, p. 572-581, 1980.

KIELHOFNER, G.; HENRY, A. D.; WALENS, D. A User's Guide to the Occupational Performance History Interview. The American Occupational Therapy Association (AOTA), 1989, 49p.

MANCINI, M. C.; COELHO, Z. A. C. Raciocínio Clínico em Terapia Ocupacional. In: DRUMOND, A. F.; REZENDE, B. M. Intervenções da Terapia Ocupacional. Belo Horizonte: Editora UFMG, 2008.

MARCOLINO, T. Q. Reflexões sobre a investigação do raciocínio clínico em terapia ocupacional em saúde mental: o caso do Método Terapia Ocupacional Dinâmica. Cad. Ter. Ocup. UFSCar, São Carlos, v. 22, n. 3, 2014, p. 635-642.

NEVES, D. A. B. Meta aprendizagem e Ciência da Informação: uma reflexão sobre o ato de aprender a aprender. Perspectivas em Ciência e Informação, v. 12, n.3, p.116-128, 2007.

ORTEGA Y GASSET, J. Meditações do Quixote. São Paulo: Livro Ibero-americano, 1967.

PEIXOTO, M. A. P.; BRANDÃO, M. A. G.; SANTOS, G. Metacognição e tecnologia educacional simbólica. Rev. Bras. Educ. Med., v.31, n.1, p. 67-80, 2007.

PINHEIRO, R. Cuidado em Saúde. In: FUNDAÇÃO OSWALDO CRUZ. Escola Politécnica de Saúde Joaquim Venâncio. Dicionário da Educação Profissional em Saúde, 2009. Disponível em: http://www.epsjv.fiocruz. br/dicionario/verbetes/cuisau.html. Acesso em: 29 dez. 2016.

RADOMSKI, M. V. Planejamento, direcionando e documentando a prática. In: RADOMSKI, M. V; TROMBLY, C. A. Terapia Ocupacional para Disfunção Física. São Paulo: Santos, 2013.

RIBEIRO, C. Metacognição: Um Apoio ao Processo de Aprendizagem. Psicologia: Reflexão e Crítica, v. 16, n.1, p. 109-116, 2003.

RUAS, T. C. B.; LEITE, F. C.; AKERMAN, M. et. al. Experiência de um estágio curricular em Terapia Ocupacional na atenção primária: foco nas necessidades em saúde infantil. ABCS Health Sci., v. 40, n. 3, p. 312-317, 2015.

SALVAdOR, C. C.; MESTRES, M. M.; GOÑI, J. O. et. al. Psicologia da Educação. Porto Alegre: Penso, 2014, 209p. 
SAND, J. The use of reflection in medical education: AMEE Guide $n^{\circ} 44$. Medical Teacher, v.31, 2009, p. 685-695.

SANTOS, G. Aprendizagem do diagnóstico fonoaudiológico clínico na perspectiva metacognitiva. Dissertação (Mestrado em Educação em Saúde) Núcleo de Tecnologia Educacional para a Saúde (NUTES) Universidade Federal do Rio de Janeiro, 2006. 190p.

SCHELL, B. B. Raciocínio Clínico: a base da prática. In: NESTAIDT, M. E; CREPEAU, E. B. Willard \& Spackman - Terapia Ocupacional. Rio de Janeiro: Guanabara Koogan, 2002, p. 80-89

SILVA, A. G.; PEIXOTO, M. A. P. Metacognição e aprendizagem do diagnóstico de enfermagem: experiências e perspectivas. Rio de Janeiro: Novas Edições Acadêmicas, 2015, 61p.

SILVA, A. G. I. PEIXOTO, M. A. P.; BRANDÃO, M. A. G.; FERREIRA, M. A.; MARTINS, J. S. A. M. Dificuldades dos estudantes de enfermagem na aprendizagem do diagnóstico de enfermagem, na perspectiva da metacognição. Esc. Anna Nery, v.15, n.3, 2011, p. 465-471.

SILVA, D. D. C.; SANTOS, I.; VARGENS, O. M. C. Metacognição como uma contribuição para as práticas educativas em enfermagem. Rev Enferm UERJ, v.23, n. 5, 2015, p.705-9.

STEDILE, N. L. R; FRIENDLANDER, M. R. Metacognição e ensino de enfermagem: uma combinação possível? Rev Latino-am Enfermagem, v.11, n. 6, 2003, p.792-799. 